\title{
ON GENERALIZED EQUILOGICAL SPACES
}

\author{
WILLIAN RIBEIRO
}

\begin{abstract}
In this paper we carry the construction of equilogical spaces into an arbitrary category $X$ topological over Set, introducing the category $X$-Equ of equilogical objects. Similar to what is done for the category Top of topological spaces and continuous functions, we study some features of the new category as (co)completeness and regular (co-)well-poweredness, as well as the fact that, under some conditions, it is a quasitopos. We achieve these various properties of the category X-Equ by representing it as a category of partial equilogical objects, as a reflective subcategory of the exact completion $\mathrm{X}_{\mathrm{ex}}$, and as the regular completion $\mathrm{X}_{\mathrm{reg}}$. We finish with examples in the particular cases, amongst others, of ordered, metric, and approach spaces, which can all be described using the $(\mathbb{T}, \mathrm{V})$-Cat setting.
\end{abstract}

\section{INTRODUCTION}

As a solution to remedy the problem of non-existence of general exponentials in Top, Scott presents first in [Sco96, and later with his co-authors Bauer and Birkedal in [BBS04, the category Equ of equilogical spaces. Formed by equipping topological $T_{0}$-spaces with arbitrary equivalence relations, Equ contains Top $_{0}$ ( $T_{0}$-spaces and continuous functions) as a full subcategory and it is cartesian closed. This fact is directly proven by showing an equivalence with the category PEqu of partial equilogical spaces, which is formed by equipping algebraic lattices with partial (not necessarily reflexive) equivalence relations. Also in [BBS04, equilogical spaces are presented as modest sets of assemblies over algebraic lattices, offering a model for dependent type theory.

Contributing to the study of Equ, a more general categorical framework, explaining why such (sub)categories are (locally) cartesian closed, was presented in [BCRS98, CR00, Ros99]. It turned out that Equ is related to the free exact completion $\left(\text { Top }_{0}\right)_{\text {ex }}$ of Top ${ }_{0}$ Car95, CM82, CV98. By the same token, suppressing the $T_{0}$-separation condition on the topological spaces, the category Equ

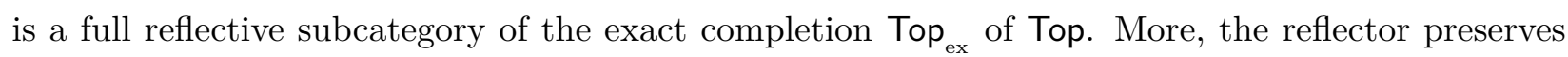
products and special pullbacks, from where it is concluded in [BCRS98] that Equ is locally cartesian closed, since Top ex $_{\text {in so }}$ BCRS98, Theorem 4.1]. It is shown in Ros98 that Equ can be presented as the free regular completion of Top [Car95, CV98], and [Men00] provides conditions for such regular completions to be quasitoposes.

2010 Mathematics Subject Classification. 54A05, 54B30, 18D15, 54D80, 18 B35.

Key words and phrases. equilogical space, topological category, exact completion, regular completion, quasitopos, $(\mathbb{T}, \mathrm{V})$-category, modest set.

Research supported by Centro de Matemática da Universidade de Coimbra - UID/MAT/00324/2013 and by the FCT PhD Grant PD/BD/128059/2016, funded by the Portuguese Government through FCT/MCTES and co-funded by the European Regional Development Fund through the Partnership Agreement PT2020. 
In this paper we start with a category $X$ and a topological functor $|-|: X \rightarrow$ Set, and, equipping each object $X$ of $X$ with an equivalence relation on its underlying set $|X|$, we define the category $\mathrm{X}$-Equ of equilogical objects and their morphisms. Recovering the results for the particular case of Top, X-Equ is (co)complete and regular (co-)well-powered. Under the hypothesis of pre-orderenrichment, we explore the concepts of separated and injective objects of $\mathbf{X}$, leading us to the definition of a category X-PEqu of partial equilogical objects. In the presence of a separation condition, we proceed presenting $\mathrm{X}$-Equ as modest sets of assemblies over injective objects; from that, we verify its properties of cartesian closedness and regularity. This is the subject of our first section.

In Section 2, analogously to the case of Top, we get similar results when considering the exact completion $X_{\text {ex }}$ and the regular completion $X_{\text {reg }}$ of $X$, culminating in the fact that $X$-Equ is a quasitopos, by the results of Men00. To do so, we use a general approach to study weak cartesian closedness of topological categories (see [CHR18]).

We finish with Section 3 , where we briefly recall the $(\mathbb{T}, V)$-Cat setting, which was introduced in [CT03] and further investigated in other papers [CH03, Hof07, and study the case when $\mathrm{X}=$ $(\mathbb{T}, \mathrm{V})$-Cat, for a suitable monad $\mathbb{T}$ and quantale $\mathrm{V}$, satisfying all conditions needed throughout the paper. Examples of such categories are Ord of preordered sets, Met of Lawvere generalized metric spaces [Law02] and App of Lowen approach spaces [Low97, amongst others. Based on full embeddings among those categories, we place full embeddings among their categories of equilogical objects.

\section{The Category of (partial) equilogical objects}

Let $\mathrm{X}$ be a category and $|-|: X \rightarrow$ Set be a topological functor. In particular $X$ is complete, cocomplete, and $|-|$ preserves both limits and colimits.

Definition 1.1. The category $X$-Equ is defined as follows.

- The objects are structures $\mathcal{X}=\left\langle X, \equiv_{|X|}\right\rangle$, where $X \in \mathrm{X}$ and $\equiv_{|X|}$ is an equivalence relation on the set $|X|$; they are called equilogical objects of $\mathrm{X}$.

- A morphism from $\mathcal{X}=\left\langle X, \equiv_{|X|}\right\rangle$ to $\mathcal{Y}=\left\langle Y, \equiv_{|Y|}\right\rangle$ is the equivalence class of a morphism $f: X \rightarrow Y$ in $X$ such that $|f|$ is an equivariant map, i.e. $x \equiv_{|X|} x^{\prime}$ implies $|f|(x) \equiv_{|Y|}|f|\left(x^{\prime}\right)$, for all $x, x^{\prime} \in|X|$, with the equivalence relation on morphisms defined by

$$
f \equiv_{\mathcal{X} \rightarrow \mathcal{Y}} g \Longleftrightarrow \forall x, x^{\prime} \in|X|,\left(x \equiv_{|X|} x^{\prime} \Longrightarrow|f|(x) \equiv_{|Y|}|g|\left(x^{\prime}\right)\right) .
$$

One can see that $\equiv_{\mathcal{X} \rightarrow \mathcal{Y}}$ is indeed an equivalence relation; reflexivity follows from the fact that the underlying maps are equivariant, symmetry and transitivity follow from the same properties for $\equiv_{|X|}$ and $\equiv_{|Y|}$.

Identity of $\mathcal{X}$ is given by $\left[1_{X}\right]$ and composition of classes $[f]: \mathcal{X} \rightarrow \mathcal{Y}$ and $[g]: \mathcal{Y} \rightarrow \mathcal{Z}$ is given by $[g] \cdot[f]=[g \cdot f]$, which is well-defined. 
Theorem 1.1. X-Equ is complete, cocomplete, regular well-powered and regular co-well-powered.

The proof of Theorem 1.1 goes along the general lines of the proof of [BBS04, Theorem 3.10]. Limits and colimits are computed in $\mathbf{X}$ and their underlying sets are endowed with appropriate equivalence relations. The properties of regular well- and regular co-well-poweredness follow from the description of equalizers and coequalizers in X-Equ.

In general X-Equ is neither well-powered nor co-well-powered, as observed in [BBS04] for topological spaces. A morphism $[m]: \mathcal{X} \rightarrow \mathcal{Y}$ is a monomorphism in X-Equ if, and only if,

$$
x \equiv_{|X|} x^{\prime} \Longleftrightarrow|m|(x) \equiv_{|Y|}|m|\left(x^{\prime}\right), \forall x, x^{\prime} \in|X|
$$

A morphism $[f]: \mathcal{X} \rightarrow \mathcal{Y}$ is an epimorphism in $\mathrm{X}$-Equ if, and only if,

$$
y \equiv_{|Y|} y^{\prime} \Longleftrightarrow \exists x, x^{\prime} \in|X| ; x \equiv_{|X|} x^{\prime} \& y \equiv_{|Y|}|f|(x) \equiv_{|Y|}|f|\left(x^{\prime}\right) \equiv_{|Y|} y^{\prime} .
$$

Having the embedding and the extension theorems configured for powersets BBS04, Theorems 3.6, 3.7], according to the authors, Scott has pointed out that those results in fact hold more generally to continuous lattices. Powersets can be generalized to algebraic lattices, and it is explained that "The reason for considering algebraic lattices is that the lattice of continuous functions between powerset spaces is not usually a powerset space, but it is an algebraic lattice. And this extends to all algebraic lattices.", culminating in the well known fact that the category ALat of algebraic lattices and Scott-continuous functions is cartesian closed GHK+80, Chapter II, Theorem 2.10].

Algebraic lattices are in particular continuous lattices, therefore injective objects in Top $_{0}$. We show below that these - injectivity and separation - are the crucial properties in order to extend the arguments of [BBS04]. Next we assume that

(a) $\mathrm{X}$ is a pre-order enriched category.

Definition 1.2. An object $X$ of $\mathrm{X}$ is said to be separated if, for each morphisms $f, g: Y \rightarrow X$ in $\mathrm{X}$, whenever $f \simeq g(f \leq g$ and $g \leq f)$, then $f=g$.

Hence an object $X$ is separated if, for each object $Y$, the pre-ordered set of morphisms $\mathrm{X}(Y, X)$ is anti-symmetric. One can check that this is equivalent to the pre-ordered set $\mathrm{X}(1, X)$ to be anti-symmetric, where $1=L\{*\}$, with $L$ : Set $\rightarrow \mathrm{X}$ the left adjoint of $|-|$.

The full subcategory $X_{\text {sep }}$ of separated objects is replete and closed under mono-sources. Since $(\operatorname{RegEpi}, \mathbb{M})$ is a factorization system for sources in the topological category $\mathbf{X}$, where $\mathbb{M}$ stands for the class of mono-sources [AHS90, Proposition 21.14], closure under mono-sources then implies that $\mathrm{X}_{\text {sep }}$ is regular epi-reflective in X [HST14, II-Proposition 5.10.1].

We will consider (pseudo-)injective objects of $X$ with respect to $|-|$-initial morphisms. Hence, denoting by $\mathrm{X}_{\mathrm{inj}}$ the full subcategory on the injectives, $Z \in \mathrm{X}_{\mathrm{inj}}$ if, and only if, for each |-|-initial morphism $y: X \rightarrow Y$ and morphism $f: X \rightarrow Z$, there exists a morphism $\hat{f}: Y \rightarrow Z$ such that 
$\hat{f} \cdot y \simeq f$

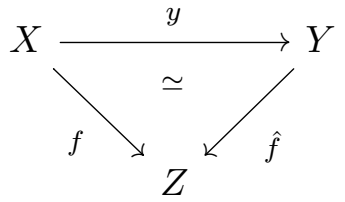

$\hat{f}$ is called an extension of $f$ along $y$; if $Z$ is separated, then $\hat{f} \cdot y=f$. Moreover, assume that

(b) for each $X, Y \in \mathrm{X}, x, x^{\prime} \in \mathrm{X}(1, X)$ and $|-|$-initial $f \in \mathrm{X}(X, Y)$,

$$
f \cdot x \simeq f \cdot x^{\prime} \Longrightarrow x \simeq x^{\prime} .
$$

Thus, for $X$ separated, if $f$ is $|-|$-initial, then $f$ is an embedding (regular monomorphism, which in our case is equivalent to $|-|$-initial with $|f|$ an injective map); hence restricting ourselves to the separated objects, injectivity with respect to |-|-initial morphisms coincides with injectivity with respect to embeddings.

Definition 1.3. The category X-PEqu of partial equilogical objects of $\mathrm{X}$ consists of:

- objects are structures $\mathcal{X}=\left\langle X, \equiv_{|X|}\right\rangle$, where $X \in \mathrm{X}_{\mathrm{inj}}$ and $\equiv_{|X|}$ is a partial (not necessarily reflexive) equivalence relation on the set $|X|$;

- a morphism from $\left\langle X, \equiv_{|X|}\right\rangle$ to $\left\langle Y, \equiv_{|Y|}\right\rangle$ is the equivalence class of an X-morphism $f: X \rightarrow Y$ such that $|f|$ is an equivariant map, with the equivalence relation on morphisms as in Definition 1.1

In order to verify an equivalence between the categories of equilogical and partial equilogical objects, we will restrict ourselves to the separated objects, so we consider now that the objects in the structures of Definitions 1.1 and 1.3 are all separated, and denote the resulting categories by $\mathrm{X}-\mathrm{Equ}_{\mathrm{sep}}$ and X-PEqu $\mathrm{sep}_{\mathrm{s}}$, respectively. We also assume that

(c) $\mathrm{X}$ has enough injectives, meaning that, for each $X \in \mathrm{X}$, there exists an $|-|-$-initial morphism $y_{X}: X \rightarrow \hat{X}$, with $\hat{X} \in \mathrm{X}_{\mathrm{inj}}$, and, if $X$ is separated, so is $\hat{X}$.

When $X$ is separated, as we have seen before, $y_{X}$ is an embedding.

Theorem 1.2. ${\mathrm{X}-\mathrm{Equ}_{\text {sep }} \text { and } \mathrm{X}-\mathrm{PEqu}}_{\mathrm{sep}}$ are equivalent.

Proof. As in the proof of [BBS04, Theorem 3.12], a functor $R: \mathrm{X}-\mathrm{PEqu}{ }_{\text {sep }} \rightarrow \mathrm{X}^{-E_{\text {Eu }}}$ sep $_{\text {is defined }}$ taking each separated partial equilogical object $\mathcal{X}$ to $R \mathcal{X}=\left\langle R X, \equiv_{|R X|}\right\rangle$, where $|R X|=\{x \in$ $\left.|X| \mid x \equiv_{|X|} x\right\}$ and $\equiv_{|R X|}$ is the restriction of $\equiv_{|X|}$. For a morphism $[f]: \mathcal{X} \rightarrow \mathcal{Y},|f|(|R X|) \subseteq|R Y|$, so we take the (co)restriction $\overline{|f|}:|R X| \rightarrow|R Y|$, lift to an X-morphism $\bar{f}: R X \rightarrow R Y$ and set $R[f]=[\bar{f}]$.

To prove that $R$ is faithful one only needs to observe that, for elements $x, x^{\prime} \in X$, if $x \equiv_{|X|} x^{\prime}$, then $x^{\prime} \equiv_{|X|} x$, and consequently $x \equiv_{|X|} x$ and $x^{\prime} \equiv_{|X|} x^{\prime}$, whence $x, x^{\prime} \in|R X|$; and to prove that $R$ is full one uses the injectivity of $Y$, providing an extension $\hat{f}: X \rightarrow Y$ of $i_{R Y} \cdot f$ along $i_{R X}$. 
Finally, for essential surjectivity let $\mathcal{X}=\left\langle X, \equiv_{|X|}\right\rangle \in \mathrm{X}-\mathrm{Equ}_{\text {sep }}$ and consider the embedding $y_{X}: X \rightarrow \hat{X}, \hat{X} \in \mathrm{X}_{\mathrm{sep}, \text { inj }}$. Endow $|\hat{X}|$ with the following partial equivalence relation

$$
\varphi \equiv_{|\hat{X}|} \psi \Longleftrightarrow \exists x, x^{\prime} \in|X| ; \varphi=\left|y_{X}\right|(x), \psi=\left|y_{X}\right|\left(x^{\prime}\right) \& x \equiv_{|X|} x^{\prime},
$$

that is, two elements of $|\hat{X}|$ are related if, and only if, they are the images by $\left|y_{X}\right|$ of elements that are related in $|X|$. The sets $|R \hat{X}|$ and $|X|$ are in bijection; using the $|-|$-initiality of $y_{X}$ and $i_{R \hat{X}}$, this bijection proves to be an isomorphism in $\mathrm{X}$, and consequently in $\mathrm{X}$-Equ $\mathrm{sep}_{\text {, }}$, by the definition of $\equiv_{|\hat{X}|}$, so $R\left\langle\hat{X}, \equiv_{|\hat{X}|}\right\rangle \cong \mathcal{X}$.

For our next result we must assume that

(d) every injective object of $\mathbf{X}$ is exponentiable.

Binary products and exponentials of injective objects are again injective, so $X_{\text {inj }}$ is a cartesian closed subcategory of $X$. We also assume that

(e) the reflector from $\mathbf{X}$ to $\mathbf{X}_{\text {sep }}$ preserves finite products;

whence the exponential of separated objects, when it exists, is again separated Day72, Sch84.

Theorem 1.3. X-PEqu ${ }_{\text {sep }}$ is cartesian closed.

Proof. Let $\mathcal{X}=\left\langle X, \equiv_{|X|}\right\rangle$ and $\mathcal{Y}=\left\langle Y, \equiv_{|Y|}\right\rangle$ be partial equilogical separated objects. We build the exponential $Y^{X}$ in $\mathrm{X}_{\text {sep,inj }}$ and endow $\left|Y^{X}\right|$ with the partial equivalence relation: $\alpha \equiv_{{ }_{\mid Y} X \mid} \beta$ if, and only if, for all $x, x^{\prime} \in X$,

$$
x \equiv_{|X|} x^{\prime} \Longrightarrow \alpha(x)=|\mathrm{ev}|(\alpha, x) \equiv_{|Y|}|\mathrm{ev}|\left(\beta, x^{\prime}\right)=\beta\left(x^{\prime}\right),
$$

for each $\alpha, \beta \in\left|Y^{X}\right|$, where ev: $Y^{X} \times X \rightarrow Y$ is the evaluation morphism in $\mathrm{X}$. Then $\mathcal{Y}^{\mathcal{X}}=$ $\left\langle Y^{X}, \equiv_{\left|{ }^{X}\right|}\right\rangle \in \mathrm{X}$-PEqu ${ }_{\text {sep }}$ and $|\mathrm{ev}|:\left|Y^{X}\right| \times|X| \rightarrow|Y|$ is equivariant, so [ev]: $\mathcal{Y}^{\mathcal{X}} \times \mathcal{X} \rightarrow \mathcal{Y}$ is a valid morphism in $X-\mathrm{PEqu}_{\text {sep }}$. More, $[\mathrm{ev}]$ satisfies the universal property: for each morphism $[f]: \mathcal{Z} \times \mathcal{X} \rightarrow \mathcal{Y}, \mathcal{Z}=\left\langle Z, \equiv_{|Z|}\right\rangle \in \mathrm{X}-$ PEqu $_{\text {sep }}$, there exists a unique $[\bar{f}]: \mathcal{Z} \rightarrow \mathcal{Y}^{\mathcal{X}}$ commuting the diagram below.

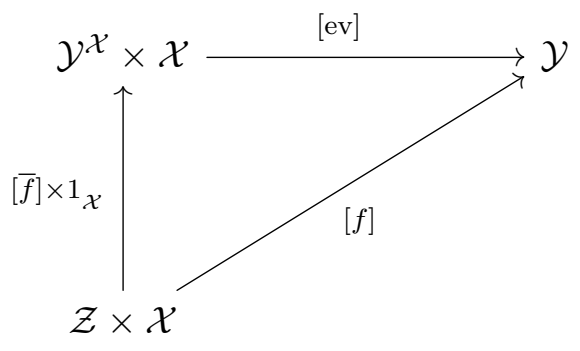

The morphism $\bar{f}: Z \rightarrow Y^{X}$ is the transpose of $f: Z \times X \rightarrow Y$, so that

$$
\mathrm{ev} \cdot\left(\bar{f} \times 1_{X}\right)=f
$$


and $[\bar{f}]$ is indeed unique, for if $\left[f^{\prime}\right]: \mathcal{Z} \rightarrow \mathcal{Y}^{\mathcal{X}}$ is such that $\left[\mathrm{ev} \cdot\left(f^{\prime} \times 1_{X}\right)\right]=[f]$, then for each $z \equiv_{|Z|} z^{\prime}$ in $Z$ and $x \equiv_{|X|} x^{\prime}$ in $X$,

$$
\begin{aligned}
\bar{f}(z)(x)=\mathrm{ev} \cdot\left(\bar{f} \times 1_{X}\right)(z, x) & =f(z, x) \equiv_{|Y|} f\left(z^{\prime}, x^{\prime}\right) \\
& \equiv_{|Y|} \mathrm{ev} \cdot\left(f^{\prime} \times 1_{X}\right)\left(z^{\prime}, x^{\prime}\right)=f^{\prime}\left(z^{\prime}\right)\left(x^{\prime}\right),
\end{aligned}
$$

hence $\bar{f}(z) \equiv_{\left|Y_{X}\right|} f^{\prime}\left(z^{\prime}\right)$, i.e. $[\bar{f}]=\left[f^{\prime}\right]$.

Therefore, by Theorem 1.2, X-Equ ${ }_{\text {sep }}$ is cartesian closed. We remark that the proof of Theorem 1.3 also applies to X-PEqu without separation.

To finish this section we discuss the presentation of equilogical objects as modest sets of assemblies, following what is done in [BBS04, Section 4].

Definition 1.4. The category of assemblies $\operatorname{Assm}\left(\mathrm{X}_{\mathrm{inj}}\right)$ over injective objects of $\mathrm{X}$ consists of the following data: objects are triples $\left(A, X, E_{A}\right)$, where $A$ is a set, $X \in \mathrm{X}_{\mathrm{inj}}$, and $E_{A}: A \rightarrow \mathcal{P}|X|$ is a function such that $E_{A}(a) \neq \emptyset$, for each $a \in A$, with $\mathcal{P}|X|$ the powerset of $|X|$. The elements of $E_{A}(a)$ are called realizers for $a$. A morphism between assemblies $\left(A, X, E_{A}\right)$ and $\left(B, Y, E_{B}\right)$ is a map $f: A \rightarrow B$ for which there exists a morphism $g: X \rightarrow Y$ in $\mathrm{X}$ such that $|g|\left(E_{A}(a)\right) \subseteq E_{B}(f(a)) ; g$ is said to be a realizer for $f$, and we say that $|g|$ tracks $f$.

Definition 1.5. An object $\left(A, X, E_{A}\right) \in \operatorname{Assm}\left(\mathrm{X}_{\mathrm{inj}}\right)$ is called a modest set if, for all $a, a^{\prime} \in A, a \neq a^{\prime}$ implies $E_{A}(a) \cap E_{A}\left(a^{\prime}\right)=\emptyset$. The full subcategory of the assemblies that are modest sets is denoted by $\operatorname{Mdst}\left(\mathrm{X}_{\mathrm{inj}}\right)$.

With these definitions, we get the same properties as those for the particular case of topological spaces, which we highlight in the following items, omitting some of the proofs that follow directly from the ones in [BBS04].

(1) $\operatorname{Mdst}\left(\mathrm{X}_{\mathrm{inj}}\right)$ and $\operatorname{Assm}\left(\mathrm{X}_{\mathrm{inj}}\right)$ have finite limits and the inclusion from modest sets to assemblies preserves them.

(2) $\operatorname{Mdst}\left(\mathrm{X}_{\mathrm{inj}}\right)$ and $\operatorname{Assm}\left(\mathrm{X}_{\mathrm{inj}}\right)$ are cartesian closed and $\operatorname{Mdst}\left(\mathrm{X}_{\mathrm{inj}}\right) \rightarrow \operatorname{Assm}\left(\mathrm{X}_{\mathrm{inj}}\right)$ preserves exponentials. For $\left(A, X, E_{A}\right)$ and $\left(B, Y, E_{B}\right)$ in $\operatorname{Assm}\left(\mathrm{X}_{\mathrm{inj}}\right)$, the exponential is $\left(C, Y^{X}, E_{C}\right)$, where $C=\{f: A \rightarrow B \mid \exists g: X \rightarrow Y \in X$ realizer for $f\}$, and $E_{C}(f)=\left\{\alpha \in\left|Y^{X}\right| \mid \alpha\right.$ tracks $\left.f\right\}$; here, for simplicity, we denote also by $\alpha$ the map from $|X|$ to $|Y|$, given by $x \mapsto|\operatorname{ev}|(\alpha, x)$, for each $x \in|X|$, where ev: $Y^{X} \times X \rightarrow Y$ is the evaluation map. If $\left(B, Y, E_{B}\right)$ is a modest set, then so is $\left(C, Y^{X}, E_{C}\right)$, for if $f, f^{\prime}: A \rightarrow B$ are tracked by $\alpha \in\left|Y^{X}\right|$, then for each $a \in A$, take $x \in E_{A}(a) \neq \emptyset$, then $\alpha(x) \in E_{B}(f(a)) \cap E_{B}\left(f^{\prime}(a)\right) \neq \emptyset$, whence $f(a)=f^{\prime}(a)$ and then $f=f^{\prime}$.

(3) $\operatorname{Mdst}\left(\mathrm{X}_{\mathrm{inj}}\right)$ is a reflective subcategory of $\mathrm{Assm}\left(\mathrm{X}_{\mathrm{inj}}\right)$.

(4) The regular subobjects of $\left(A, X, E_{A}\right)$ in $\operatorname{Assm}\left(\mathrm{X}_{\mathrm{inj}}\right)$, or in $\mathrm{Mdst}\left(\mathrm{X}_{\mathrm{inj}}\right)$, are in bijective correspondence with the powerset of $A$. 
(5) $\operatorname{Mdst}\left(\mathrm{X}_{\mathrm{inj}}\right)$ and $\operatorname{Assm}\left(\mathrm{X}_{\mathrm{inj}}\right)$ are regular categories.

Theorem 1.4. X-PEqu and $\mathrm{Mdst}\left(\mathrm{X}_{\mathrm{inj}}\right)$ are equivalent.

Proof. Define the functor $F: \operatorname{Mdst}\left(\mathrm{X}_{\text {inj }}\right) \rightarrow \mathrm{X}$-PEqu assigning to $\left(A, X, E_{A}\right)$ the object $\left\langle X, \equiv_{|X|}\right\rangle$, where

$$
x \equiv_{|X|} x^{\prime} \Longleftrightarrow \exists a \in A ; x, x^{\prime} \in E_{A}(a),
$$

and on morphisms $F$ assigns to each $f:\left(A, X, E_{A}\right) \rightarrow\left(B, Y, E_{B}\right)$ the class of a realizer $g: X \rightarrow Y$ for $f ; \equiv_{|X|}$ is indeed an equivalence relation and two realizers for $f$ are in the same equivalence class, so $F$ is well-defined.

Faithfulness of $F$ follows from the observation in item (2) above: two maps tracked by the same realizer must be equal. To see that $F$ is full, take a morphism $[g]: F\left(A, X, E_{A}\right) \rightarrow F\left(B, Y, E_{B}\right)$ in X-PEqu. For each $a \in A$, let $x \in E_{A}(a) \neq \emptyset$; then $x \equiv_{|X|} x$ and so $|g|(x) \equiv_{|Y|}|g|(x)$, that is, there exists $b \in B$ such that $|g|(x) \in E_{B}(b)$, whence we set $f(a)=b$; this $b$ is uniquely determined since $\left(B, Y, E_{B}\right)$ is a modest set, therefore we have a map $f: A \rightarrow B$, which, by definition, has $g$ as a realizer.

Now let $\left\langle X, \equiv_{|X|}\right\rangle$ be a partial equilogical object and define $\left(A, X, E_{A}\right)$ by $A=\left\{x \in|X| \mid x \equiv_{|X|}\right.$ $x\} / \equiv_{|X|}$ and $E_{A}([x])=[x] \subseteq \mathcal{P}|X|$. Hence $F\left(A, X, E_{A}\right)=\left\langle X, \equiv_{|X|}\right\rangle$ and $F$ is essentially surjective.

The same argument can be repeated replacing $X_{\text {inj }}$ with $X_{\text {sep, inj }}$, so we obtain $\operatorname{Mdst}\left(X_{\text {sep, inj }}\right) \cong X$ $\mathrm{PEqu}_{\mathrm{sep}} \cong \mathrm{X}-\mathrm{Equ}_{\mathrm{sep}}$. Properties from items (1) to (5) remain valid, so they also hold for $\mathrm{X}$-Equ $\mathrm{sep}_{\text {. }}$.

\section{EQUilogicAl OBJECTS AND EXACT COMPLETION}

The category Equ of equilogical spaces can also be obtained as a full reflective subcategory of the exact completion [BCRS98, Car95, CM82] Top ${ }_{\text {ex }}$ of the category of topological spaces [Ros99], and this is a particular instance of a general process to obtain such categories [BCRS98].

We can describe the exact completion $\mathrm{X}_{\mathrm{ex}}$ of $\mathrm{X}$ as: objects are pseudo-equivalence relations on $\mathrm{X}$, that is, parallel pairs of morphisms $X_{1} \underset{r_{2}}{\stackrel{r_{1}}{\longrightarrow}} X_{0}$ of $\mathrm{X}$ satisfying

(i) reflexivity: there exists a morphism $r: X_{0} \rightarrow X_{1}$ such that $r_{1} \cdot r=1_{X_{0}}=r_{2} \cdot r$;

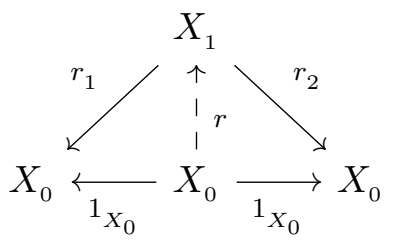


(ii) symmetry: there exists a morphism $s: X_{1} \rightarrow X_{1}$ such that $r_{1} \cdot s=r_{2}$ and $r_{2} \cdot s=r_{1}$;

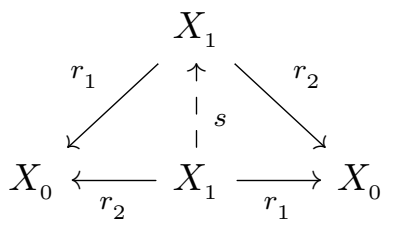

(iii) transitivity: for $r_{3}, r_{4}: X_{2} \rightarrow X_{1}$ a pullback of $r_{1}, r_{2}$, there exists a morphism $t: X_{2} \rightarrow X_{1}$ commuting the following diagram

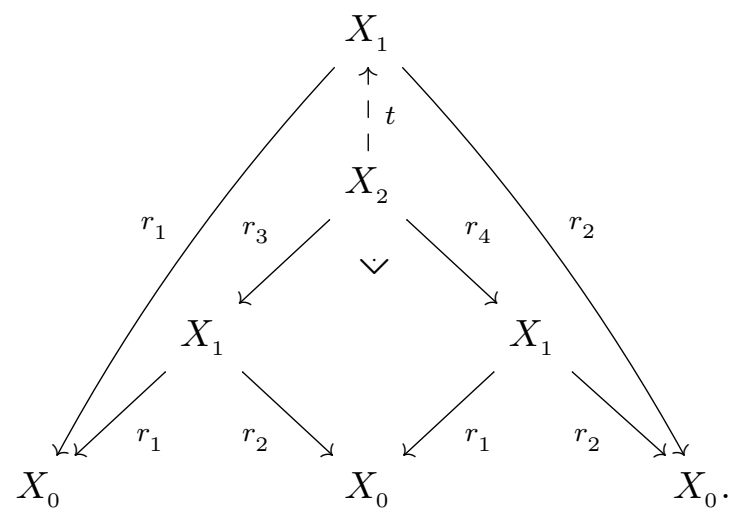

A morphism from $X_{1} \underset{r_{2}}{\stackrel{r_{1}}{\longrightarrow}} X_{0}$ to $Y_{1} \underset{s_{2}}{\stackrel{s_{1}}{\longrightarrow}} Y_{0}$ is an equivalence classe $[f]$ of an $\mathrm{X}$-morphism $f: X_{0} \rightarrow Y_{0}$ such that there exists $g: X_{1} \rightarrow Y_{1}$ in X satisfying $f \cdot r_{i}=s_{i} \cdot g, i=1,2$.

$$
\begin{gathered}
X_{1}-\stackrel{g}{-} \rightarrow Y_{1} \\
r_{1} \downarrow \downarrow r_{2} \quad s_{1} \downarrow \downarrow s_{2} \\
X_{0} \longrightarrow Y_{0} .
\end{gathered}
$$

Here two morphisms $f_{1}, f_{2}: X_{0} \rightarrow Y_{0}$ are related if, and only if, there exists a morphism $h: X_{0} \rightarrow Y_{1}$ such that $f_{i}=s_{i} \cdot h, i=1,2$.

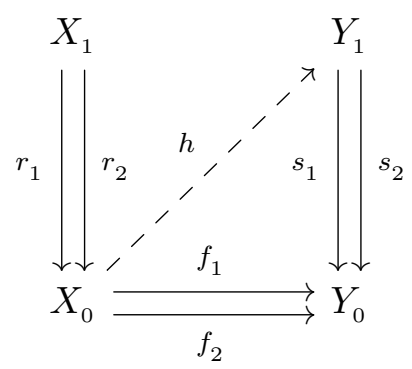

Since it is topological over Set, $\mathrm{X}$ has a stable factorization system for morphisms given by (Epi, RegMono) [AHS90, Remark 15.2(3), Proposition 21.14] (see also [CHR18]). Let us denote by $\operatorname{PER}\left(\mathbf{X}\right.$, RegMono) the full subcategory of $\mathbf{X}_{\text {ex }}$ of the pseudo-equivalence relations $X_{1} \underset{r_{2}}{\stackrel{r_{1}}{\rightleftarrows}} X_{0}$ such that $\left\langle r_{1}, r_{2}\right\rangle: X_{1} \rightarrow X_{0} \times X_{0}$ is a regular monomorphism.

Lemma 2.1. X-Equ and $\operatorname{PER}(\mathrm{X}, \mathrm{RegMono})$ are equivalent. 
Proof. For each equilogical object $\left\langle X, \equiv_{|X|}\right\rangle$, consider $E_{X}=\left\{\left(x, x^{\prime}\right) \in|X| \times|X| \mid x \equiv_{|X|} x^{\prime}\right\}$ and the source $\left(\pi_{i}^{X}: E_{X} \rightarrow|X|\right)_{i=1,2}$ of the projections from $E_{X}$ onto $|X|$. Take its |-|-initial lifting, which by abuse of notation we denote by $\left(\pi_{i}^{X}: E_{X} \rightarrow X\right)_{i=1,2}$. Hence $E_{X} \underset{\pi_{2}^{X}}{\stackrel{\pi_{1}^{X}}{\longrightarrow}} X$ belongs to $\operatorname{PER}(\mathrm{X}, \mathrm{RegMono})$ and each morphism $[f]: \mathcal{X} \rightarrow \mathcal{Y}$ in $\mathrm{X}$-Equ is a valid morphism

$$
[f]:\left(E_{X}, X, \pi_{1}^{X}, \pi_{2}^{X}\right) \rightarrow\left(E_{Y}, Y, \pi_{1}^{Y}, \pi_{2}^{Y}\right)
$$

in $\operatorname{PER}(\mathrm{X}, \mathrm{RegMono})$.

That correspondence defines a functor which is fully faithful and, for a pseudo-equivalence relation $X_{1} \underset{r_{2}}{\stackrel{r_{1}}{\longrightarrow}} X_{0}$ in $\operatorname{PER}(\mathrm{X}, \operatorname{RegMono})$, define the equilogical object $\left\langle X_{0}, \equiv_{\left|X_{0}\right|}\right\rangle$ by

$$
x_{0} \equiv_{\left|X_{0}\right|} x_{0}^{\prime} \Longleftrightarrow\left(\exists \text { (unique) } x_{1} \in X_{1}\right)\left|r_{1}\right|\left(x_{1}\right)=x_{0} \&\left|r_{2}\right|\left(x_{1}\right)=x_{0}^{\prime},
$$

for each $x_{0}, x_{0}^{\prime} \in X_{0}$. Then $E_{X_{0}} \underset{\pi_{2}^{X_{0}}}{\stackrel{\pi_{1}^{X_{0}}}{\longrightarrow}} X_{0}$ is isomorphic to $X_{1} \underset{r_{2}}{\stackrel{r_{1}}{\longrightarrow}} X_{0}$ in PER(X, RegMono) and the functor is essentially surjective.

Hence [BCRS98, Theorem 4.3] states that

Theorem 2.1. $\mathrm{X}$-Equ $\cong \mathrm{PER}(\mathrm{X}, \mathrm{RegMono})$ is a full reflective subcategory of $\mathrm{X}_{\mathrm{ex}}$; the reflector preserves finite products and commutes with change of base in the codomain.

Next we wish to prove that $\mathrm{X}$-Equ is cartesian closed, so by Theorem 2.1] and [Sch84, Theorem $1.2]$, it suffices to show that $X_{\text {ex }}$ is cartesian closed. To do so, we will apply the following result derived from [Ros99, Theorem 1, Lemma 4] (see also [CHR18, Theorem 1.1]).

Theorem 2.2. Let $\mathrm{X}$ be a complete, infinitely extensive and well-powered category with factorizations (RegEpi, Mono) such that $f \times 1$ is an epimorphism whenever $f$ is a regular epimorphism. Then $\mathrm{X}_{\mathrm{ex}}$ is cartesian closed provided $\mathrm{X}$ is weakly cartesian closed.

Since $\mathbf{X}$ is topological over Set, in order to use the latter theorem, we will assume that

(f) $\mathrm{X}$ is infinitely extensive;

more, assuming also conditions (a) to (e) from the previous section, following the same steps of the proofs of [CHR18, Theorems 5.3 and 5.5], we deduce the following result.

Proposition 2.1. $\mathrm{X}$ is weakly cartesian closed.

Furthermore, we can verify that $X_{e x}$ is actually locally cartesian closed. Consider the restriction functor $|-|: X_{\mathrm{inj}} \rightarrow \mathrm{Pfn}$, where $\mathrm{Pfn}$ is the category of sets and partial functions. The category $\mathcal{F}\left(\mathrm{X}_{\mathrm{inj}}, \mid-\right.$ $\mid$ ), or simply $\mathcal{F}\left(\mathrm{X}_{\text {inj }}\right)$, is described in [CR00] as follows: objects are triples $(X, A, \sigma: A \rightarrow|X|)$, where 
$X$ is an injective object of $\mathrm{X}, A$ is a set and $\sigma$ is a function; a morphism $f:(X, A, \sigma: A \rightarrow|X|) \rightarrow$ $(Y, B, \delta: B \rightarrow|Y|)$ is a map $f: A \rightarrow B$ such that there exists $g: X \rightarrow Y$ in $\mathrm{X}$ commuting the diagram

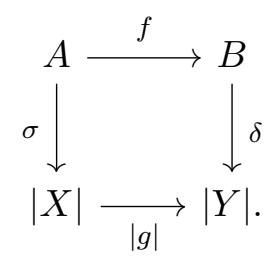

Proposition 2.2. The categories $\mathrm{X}$ and $\mathcal{F}\left(\mathrm{X}_{\mathrm{inj}}\right)$ are equivalent.

Proof. Define the functor $G: \mathrm{X} \rightarrow \mathcal{F}\left(\mathrm{X}_{\mathrm{inj}}\right)$ by

$$
G X=\left(\hat{X},|X|, \sigma_{X}=\left|y_{X}\right|:|X| \rightarrow|\hat{X}|\right)
$$

where $y_{X}$ is the $|-|$-initial morphism assured by condition (c) in the previous section; for each morphism $f: X \rightarrow Y$, injectivity of $\hat{Y}$ implies the existence of a morphism $g: \hat{X} \rightarrow \hat{Y}$ extending $y_{Y} \cdot f$ along $y_{X}$

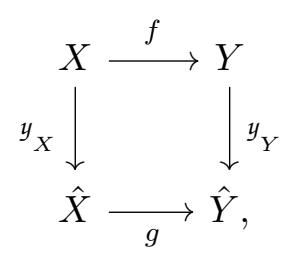

hence we set $G f=|f|$. $G$ is faithful and to see it is full, let $f:|X| \rightarrow|Y|$ be a map commuting the diagram

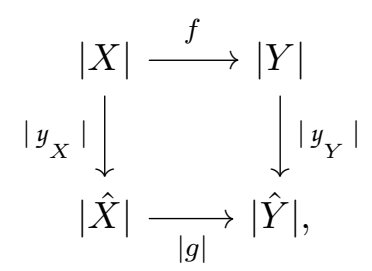

for some $g: \hat{X} \rightarrow \hat{Y}$ in $\mathrm{X}$, then |-|-initiality of $y_{Y}$ implies the existence of a unique $\bar{f}: X \rightarrow Y$ such that $G \bar{f}=|\bar{f}|=f$.

For essential surjectivity, let $(X, A, \sigma: A \rightarrow|X|)$ in $\mathcal{F}\left(\mathrm{X}_{\text {inj }}\right)$ and take the $|-|$-initial lifting of $\sigma$, that we denote by $\sigma_{\mathrm{ini}}: A_{\mathrm{ini}} \rightarrow X$, so $\left|A_{\mathrm{ini}}\right|=A$ and $\left|\sigma_{\mathrm{ini}}\right|=\sigma$. Hence $G A_{\mathrm{ini}}=\left(\hat{A}_{\mathrm{ini}}, A,\left|y_{A_{\mathrm{ini}}}\right|: A \rightarrow\left|\hat{A}_{\mathrm{ini}}\right|\right)$ and we verify that the identity map $1_{A}: A \rightarrow A$ is a morphism from $(X, A, \sigma)$ to $\left(\hat{A}_{\text {ini }}, A,\left|y_{A_{\text {ini }}}\right|\right)$, and vice-versa. The latter fact comes readly from injectivity of $\hat{A}_{\text {ini }}$ and |-|-initiality of $\sigma_{\text {ini }}$ :

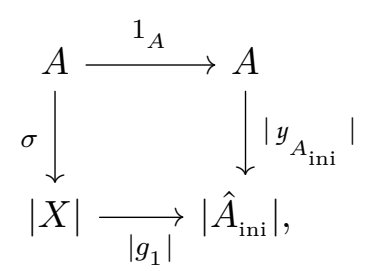


for some morphism $g_{1}: X \rightarrow \hat{A}_{\text {ini }}$, and by injectivity of $X$ and $|-|$-initiality of $y_{A_{\mathrm{ini}}}$ :

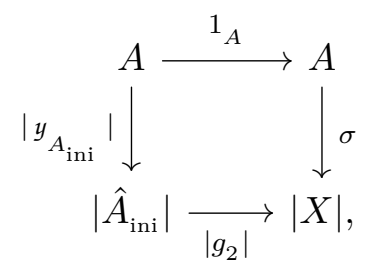

for some morphism $g_{2}: \hat{A}_{\mathrm{ini}} \rightarrow X$. Therefore, $G A_{\mathrm{ini}} \cong(X, A, \sigma: A \rightarrow|X|)$ in $\mathcal{F}\left(\mathrm{X}_{\mathrm{inj}}\right)$.

Since $\mathrm{X}_{\mathrm{inj}}$ is (weakly) cartesian closed, as shown in [CR00], $\mathrm{X} \cong \mathcal{F}\left(\mathrm{X}_{\mathrm{inj}}\right)$ has all weak simple products (in particular it is weakly cartesian closed), and more, $X \cong \mathcal{F}\left(\mathrm{X}_{\mathrm{inj}}\right)$ is weakly locally cartesian closed, i.e. it has weak dependent products, whence by [CR00, Theorem 3.3], $\mathrm{X}_{\mathrm{ex}} \cong$ $\mathcal{F}\left(\mathrm{X}_{\mathrm{inj}}\right)_{\text {ex }}$ is locally cartesian closed.

Therefore, by Theorem 2.1, we conclude that X-Equ is locally cartesian closed (see for instance [HST14, III-Corollary 4.6.2]), and, being complete and cocomplete, one may ask whether this category is actually a quasitopos.

As discussed in Ros98, “... the full subcategory of $\mathcal{C}_{\mathrm{ex}}$ consisting of those equivalence spans which are kernel pairs in $\mathcal{C}$ gives the free regular completion $\mathcal{C}_{\text {reg }}$ of $\mathcal{C}$.", where in that context equivalence span means pseudo-equivalence relation. Hence, similar to what is observed in [Men00] for topological spaces, the category $\mathrm{X}$-Equ, presented by $\operatorname{PER}(\mathrm{X}, \mathrm{RegMono})$, is equivalent to the regular completion $\mathrm{X}_{\text {reg }}$ of $\mathrm{X}$.

It is easy to depict the latter equivalence using the classical description of $X_{\text {reg }}$ Car95: objects are $\mathrm{X}$-morphisms $f: X \rightarrow Y$, and a morphism from $f: X \rightarrow Y$ to $g: Z \rightarrow W$ is an equivalence class $[l]$ of an X-morphism $l: X \rightarrow Z$ such that $g \cdot l \cdot f_{0}=g \cdot l \cdot f_{1}$, where $f_{0}, f_{1}$ form the kernel pair of $f$.

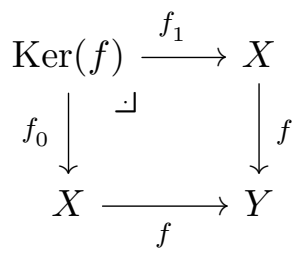

Two such arrows $l$ and $m$ are equivalent if $g \cdot l=g \cdot m$.

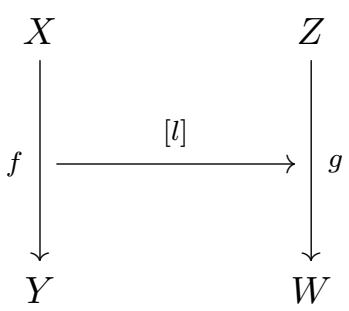

Lemma 2.2. $\mathrm{X}_{\text {reg }}$ and $\operatorname{PER}(\mathrm{X}, \mathrm{RegMono})$ are equivalent. 
Proof. Define $F: \mathrm{X}_{\text {reg }} \rightarrow \operatorname{PER}(\mathrm{X}, \mathrm{RegMono})$ as in the diagram below,

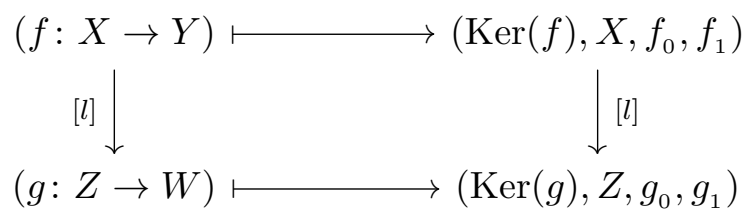

so it is a well-defined functor, since $l: X \rightarrow Z$ satisfies $g \cdot l \cdot f_{0}=g \cdot l \cdot f_{1}$ if, and only if, there exists a unique $\bar{l}: \operatorname{Ker}(f) \rightarrow \operatorname{Ker}(g)$ such that $g_{0} \cdot \bar{l}=l \cdot f_{0}$ and $g_{1} \cdot \bar{l}=l \cdot f_{1}$.

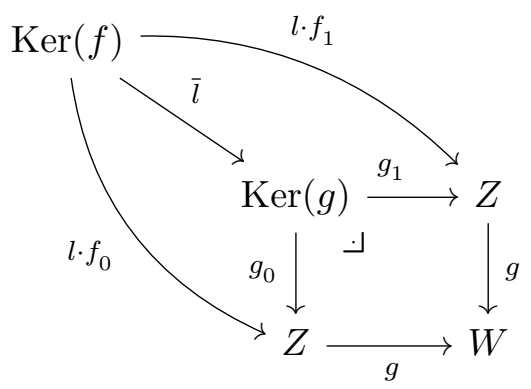

Then $F$ is fully faithful, and it is essentially surjective because each pseudo-equivalence relation $X_{1} \underset{r_{2}}{\stackrel{r_{1}}{\longrightarrow}} X_{0}$ with $\left\langle r_{1}, r_{2}\right\rangle: X_{1} \rightarrow X_{0} \times X_{0}$ a regular monomorphism is seen to form the kernel pair of the $|-|$-final lifting $\bar{p}: X_{0} \rightarrow \tilde{X}_{0}$ of the projection map $p:\left|X_{0}\right| \rightarrow\left|X_{0}\right| / \sim$, where $\sim$ is the equivalence relation on $\left|X_{0}\right|$ defined in the proof of Lemma 2.1 .

We now intend to apply [Men00, Corollary 8.4.2]; by condition (f) and Proposition 2.2. $\mathrm{X}$ is an (infinitely) extensive weakly locally cartesian closed category, hence we are only missing the chaotic situation described right after [Men00, Lemma 7.3.3]. This comes from the observation that the topos Set is a mono-localization of $\mathrm{X}$, since the topological functor $|-|: X \rightarrow$ Set is faithful, preserves finite limits and has a full embedding as a right adjoint [AHS90, Proposition 21.12]. Therefore, by Lemma 2.1. Lemma 2.2 and [Men00, Corollary 8.4.2], we conclude:

Theorem 2.3. X-Equ is a quasitopos.

\section{The Case $\mathrm{X}=(\mathbb{T}, \mathrm{V})$-Cat}

We briefly introduce the (T,, $\mathrm{V})$-Cat setting, and refer the reader to the reference CT03] for details (see also [HST14).

Although introduced in a more general setting, we are interested here in the case when - $\mathrm{V}=(\mathrm{V}, \otimes, k)$ is a commutative unital quantale (see for instance [HST14, II-Section 1.10]) which is also a Heyting algebra (so that the operation infimum $\wedge$ also has a right adjoint), and - $\mathbb{T}=(T, m, e):$ Set $\rightarrow$ Set is a monad satisfying the Beck-Chevalley condition ( $T$ preserves weak pullbacks and the naturality squares of $m$ are weak pullbacks [CHJ14]) that is laxly extended to the pre-ordered category $\mathrm{V}$-Rel, which has as objects sets and as morphisms $\mathrm{V}$-relations $r: X \rightarrow Y$, i.e. V-valued maps $r: X \times Y \rightarrow \mathrm{V}$. 
Hence we assume that there exists a functor $T: \mathrm{V}$-Rel $\rightarrow \mathrm{V}$-Rel extending $T$, by abuse of notation denoted by the same letter, that commutes with involution: $T\left(r^{\circ}\right)=(T r)^{\circ}$, for each $r: X \rightarrow Y \in$ V-Rel, where $r^{\circ}(y, x)=r(x, y)$, for each $(x, y) \in X \times Y$.

The functor $T$ turns $m$ and $e$ into oplax transformations, meaning that the naturality diagrams become:

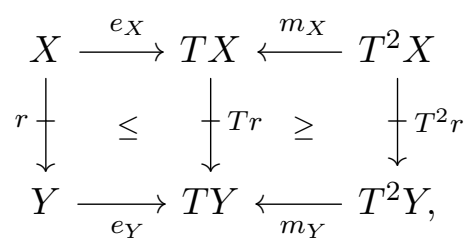

for each $\mathrm{V}$-relation $r: X \mapsto Y$.

Hence we have a lax monad on $\mathrm{V}$-Rel $[\mathrm{CH} 04]$ and $(\mathbb{T}, \mathrm{V})$-Cat is defined as the category of EilenbergMoore lax algebras for that lax monad: objects are pairs $(X, a)$, where $X$ is a set and $a: T X \mapsto X$ is a $\mathrm{V}$-relation, which is reflexive and transitive.

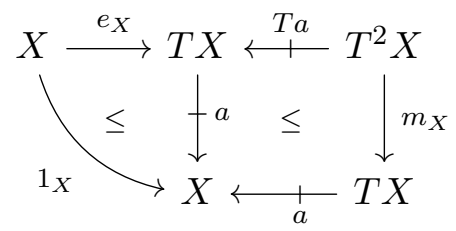

Such pairs are called $(\mathbb{T}, \bigvee)$-categories; a morphism from $(X, a)$ to $(Y, b)$ is a map $f: X \rightarrow Y$ commuting the diagram below.

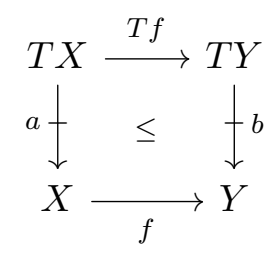

Such a map is called $(\mathbb{T}, \mathrm{V})$-functor.

We are also going to restrict ourselves to the case that the extension $T$ to $\mathrm{V}$-Rel is determined by a $\mathbb{T}$-algebra structure map $\xi: T V \rightarrow V$, so we are in the setting of topological theories [Hof07]. (see also [CT14]), hence $\vee$ has a $(\mathbb{T}, \bigvee)$-category structure given by the composite

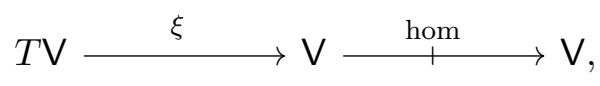

where hom: $\mathrm{V} \times \mathrm{V} \rightarrow \mathrm{V}$ is the left adjoint of $\otimes$, so

$$
u \otimes v \leq w \Longleftrightarrow u \leq \operatorname{hom}(v, w)
$$

for each $u, v, w$ in the quantale $\mathrm{V}$.

The forgetful functor $|-|:(\mathbb{T}, V)-C a t ~ \rightarrow$ Set is topological [CH03, CT03], and before we provide examples of categories given by $(\mathbb{T}, \bigvee)$-Cat, we verify that, for suitable monad $\mathbb{T}$ and quantale $\mathrm{V}$ satisfying the conditions assumed so far in this section, $(\mathbb{T}, \bigvee)$-Cat satisfies all conditions (a) to (f) 
from the two previous sections. In each item, we highlight the properties that are needed in order to achieve the respective condition, adding the references where that is proved.

(a) $(\mathbb{T}, \mathrm{V})$-Cat is pre-ordered enriched. For $(\mathbb{T}, \mathrm{V})$-categories $(X, a)$ and $(Y, b)$, consider the following relation on the set of $(\mathbb{T}, \mathrm{V})$-functors from $(X, a)$ to $(Y, b)$ :

$$
f \leq g \Longleftrightarrow \forall x \in X, k \leq b\left(e_{Y}(f(x)), g(x)\right)
$$

This determines a pre-order, first defined in [CT03, which is compatible with composition of (T, V)functors. One can also check that a $(\mathbb{T}, \mathrm{V})$-category $(X, a)$ is separated if, and only if, the following pre-order on $X$ is anti-symmetric:

$$
x \leq x^{\prime} \Longleftrightarrow k \leq a\left(e_{X}(x), x^{\prime}\right)
$$

(see [HST14, III-Proposition 3.3.1]).

(b) |-|-initial $(\mathbb{T}, \mathrm{V})$-functors reflect the order. Let $(X, a),(Y, b)$ be $(\mathbb{T}, \mathrm{V})$-categories, $x, x^{\prime}: 1 \rightarrow$ $(X, a)$ be $(\mathbb{T}, V)$-functors, where $1=\left(\{*\}, e_{\{*\}}^{\circ}\right)$ (the discrete structure on the singleton [HST14, IIISection 3.2]), and $f:(X, a) \rightarrow(Y, b)$ an $|-|$-initial $(\mathbb{T}, \mathrm{V})$-functor such that $f \cdot x \simeq f \cdot x^{\prime} ;|-|$-initiality of $f$ means that $a(\mathfrak{x}, x)=b(T f(\mathfrak{x}), f(x))$, for each $\mathfrak{x} \in T X, x \in X$. We calculate:

$$
\begin{aligned}
k & \leq b\left(e_{Y}(f \cdot x(*)), f \cdot x^{\prime}(*)\right) & & \left(\text { definition of } f \cdot x \leq f \cdot x^{\prime}\right) \\
& \leq b\left(T f \cdot e_{X}(x(*)), f \cdot x^{\prime}(*)\right) & & (\text { composition is associative, } e \text { is natural }) \\
& \leq a\left(e_{X}(x(*)), x^{\prime}(*)\right) & & (f \text { is }|-| \text {-initial }),
\end{aligned}
$$

so $x \leq x^{\prime}$ and in the same fashion $x^{\prime} \leq x$, thus $x \simeq x^{\prime}$.

(c) $(\mathbb{T}, \bigvee)$-Cat has enough injectives. The tensor product $\otimes$ of $\bigvee$ induces a functor $\otimes:(\mathbb{T}, \bigvee)$-Cat $\times$ $(\mathbb{T}, \mathrm{V})$-Cat $\rightarrow(\mathbb{T}, \mathrm{V})$-Cat, with

$$
(X, a) \otimes(Y, b)=(X \times Y, c),
$$

where, for each $\mathfrak{w} \in T(X \times Y),(x, y) \in X \times Y$,

$$
c(\mathfrak{w},(x, y))=a\left(T \pi_{X}(\mathfrak{w}), x\right) \otimes b\left(T \pi_{Y}(\mathfrak{w}), y\right),
$$

and $\pi_{X}, \pi_{Y}$ are the projections from $X \times Y$ onto $X$ and $Y$, respectively. The following facts can be found in [CH09, Hof11, CCH15]: for each $(\mathbb{T}, \mathrm{V})$-category $(X, a), a: T X \rightarrow X$ defines a (T, $\mathrm{V})$ functor

$$
a: X^{\mathrm{op}} \otimes X \rightarrow \mathrm{V}
$$

where $X^{\mathrm{op}}=\left(T X, m_{X} \cdot(T a)^{\circ} \cdot m_{X}\right)$; the $\otimes$-exponential mate $y_{X}: X \rightarrow \mathrm{V}^{\mathrm{op}}$ of $a$ is fully faithful; the $(\mathbb{T}, \mathrm{V})$-category $P X=\mathrm{V}^{X^{\mathrm{op}}}$ is injective and if $(X, a)$ is separated, so is $P X$. 
(d) Injectives are exponentiable. Conditions under which injectivity implies exponentiability in $(\mathbb{T}, \mathrm{V})$-Cat are studied in [CHR18. We recall them next. Consider the maps

$$
\mathrm{V} \otimes \mathrm{V} \stackrel{\otimes}{\longrightarrow} \mathrm{V} \text { and } X \stackrel{(-, u)}{\longrightarrow} X \otimes \mathrm{V}
$$

with $\left(\mathrm{V}, \operatorname{hom}_{\xi}\right),(X, a) \in(\mathbb{T}, \mathrm{V})$-Cat. Define also for a $\mathrm{V}$-relation $r: X \rightarrow Y$ and $u \in \mathrm{V}$, the $\mathrm{V}$ relation $r \otimes u: X \rightarrow Y$ given by

$$
(r \otimes u)(x, y)=r(x, y) \otimes u
$$

for each $(x, y) \in X \times Y$. As a final condition, assume that, for all $u, v, w \in \mathrm{V}$,

$$
w \wedge(u \otimes v)=\left\{u^{\prime} \otimes v^{\prime} \mid u^{\prime} \leq u, v^{\prime} \leq v, u^{\prime} \otimes v^{\prime} \leq w\right\}
$$

which is equivalent to exponentiability of injective V-categories (see [HR13, Theorem 5.3]). Then [CHR18, Theorem 5.4] says the following:

Theorem 3.1. Suppose that: the maps $\otimes$ and $(-, u)$ in $(1)$ are $(\mathbb{T}, \vee)$-functors; for every injective $(\mathbb{T}, \mathrm{V})$-category $(X, a)$ and every $u \in \mathrm{V}, T(a \otimes u)=T a \otimes u$, with those $\mathrm{V}$-relations defined as in (2); and (3) holds. Then every injective $(\mathbb{T}, \mathrm{V})$-category is exponentiable in $(\mathbb{T}, \mathrm{V})$-Cat.

(e) The reflector from $(\mathbb{T}, \mathrm{V})$-Cat to $(\mathbb{T}, \mathrm{V})-\mathrm{Cat}_{\mathrm{sep}}$ preserves finite products. This is proved in [CHR18, Proposition 5.4].

(f) $(\mathbb{T}, \mathrm{V})$-Cat is infinitely extensive. This is proved in MST06] under the condition that $T$ is a taut functor [Man02, what comes for free from the assumption that $T$ preserves weak pullbacks.

To give examples of categories satisfying all the conditions above, we consider:

- the identity monad $\mathbb{I}=(\mathrm{Id}, 1,1)$ on Set laxly extended to the identity lax monad on $\mathrm{V}$-Rel;

- the ultrafilter monad $\mathbb{U}$ with the Barr extension to V-Rel [HST14, IV-Corollary 2.4.5], with $\mathrm{V}$ integral and completely distributive (see, for instance, [HST14, II-Section 1.11]);

- the list monad (or free monoid monad) $\mathbb{L}=(L, m, e)$ (see [HST14, II-Examples 3.1.1(2)]), with the extension $L: \mathrm{V}$-Rel $\rightarrow \mathrm{V}$-Rel that sends each $r: X \rightarrow Y$ to the $\mathrm{V}$-relation $L r: L X \rightarrow L Y$ given by

$$
\operatorname{Lr}\left(\left(x_{1}, \ldots, x_{n}\right),\left(y_{1}, \ldots, y_{m}\right)\right)=\left\{\begin{array}{l}
r\left(x_{1}, y_{1}\right) \otimes \cdots \otimes r\left(x_{n}, y_{n}\right), \text { if } n=m \\
\perp, \text { if } n \neq m
\end{array}\right.
$$

- the monad $\mathbb{M}=(-\times M, m, e)$, for a monoid $\left(M, \cdot 1_{M}\right)$, with $m_{X}: X \times M \times M \rightarrow X \times M$ given by $m_{X}(x, a, b)=(x, a \cdot b)$ and $e_{X}: X \rightarrow X \times M$ given by $e_{X}(x)=\left(x, 1_{M}\right)$ (see [HST14, V-Section 1.4]). The extension $-\times M: \mathrm{V}$-Rel $\rightarrow \mathrm{V}$-Rel sends the $\mathrm{V}$-relation $r: X \rightarrow Y$ to the $\mathrm{V}$-relation 
$r \times M: X \times M \mapsto Y \times M$ with

$$
r \times M((x, a),(y, b))=\left\{\begin{array}{l}
r(x, y), \text { if } a=b, \\
\perp, \text { if } a \neq b .
\end{array}\right.
$$

As well as the quantales: $2=(\{\perp, \top\}, \wedge, \top), \mathrm{P}_{+}=\left([0, \infty]^{\mathrm{op}},+, 0\right), \mathrm{P}_{\max }=\left([0, \infty]^{\mathrm{op}}, \max , 0\right)$, $2^{2}=(\{\perp, u, v, \top\}, \wedge, \top)$ (the diamond lattice [HST14, II-Exercise 1.H]) and $\Delta$ (the quantale of distribution functions [HR13]). We assemble the table:

\begin{tabular}{|l|c|c|c|c|}
\hline $\mathbb{V}$ & $\mathbb{I}$ & $\mathbb{U}$ & $\mathbb{L}$ & $\mathbb{M}$ \\
\hline 2 & Ord & Top & MultiOrd & $(\mathbb{M}, 2)$-Cat \\
\hline$P_{+}$ & Met & App & & \\
\hline$P_{\max }$ & UltMet & NA-App & & \\
\hline $2^{2}$ & BiRel & BiTop & & \\
\hline$\Delta$ & ProbMet & & & \\
\hline
\end{tabular}

- Ord is the category of pre-ordered spaces,

- Met is the category of Lawvere generalized metric spaces [Law02,

- UltMet is the full subcategory of Met of ultra-metric spaces [HST14, III-Exercise 2.B],

- BiRel is the one of sets and birelations [HST14, III-Examples 1.1.1(3)],

- ProbMet is the category of probabilistic metric spaces [HR13],

- Top is the usual category of topological spaces and continuous functions,

- App is that of Lowen's approach spaces [Low97, and

- NA-App is the full subcategory of App of non-Archimedean approach spaces studied in details in [CVO17], and denoted in [Hof14] by UApp,

- BiTop is the category of bitopological spaces and bicontinuous maps [HST14, III-Exercise 2.D],

- MultiOrd is the category of multi-ordered sets [HST14, V-Section 1.4], and

- (M,2)-Cat can be interpreted as the category of $M$-labelled ordered sets [HST14, V-Section 1.4]. For instance, an object of Ord-Equ is a pre-ordered set $(X, \leq)$ together with an equivalence relation $\equiv_{X}$ on $X$; separatedness of $(X, \leq)$ means that $\leq$ is anti-symmetric, so the objects of Ord-Equ ${ }_{\text {sep }}$ are partially ordered sets equipped with equivalence relations on their underlying sets. Further, a partial equilogical separated object in Ord-PEqu ${ }_{\text {sep }}$ is a complete lattice (injective ordered set) together with an equivalence relation on the underlying set. In the same fashion, the objects of the category $\operatorname{Mdst}\left(\operatorname{Ord}_{\text {sep,inj }}\right)$ are triples $\left(A,(X, \leq), E_{A}\right)$, with $A$ a set, $E_{A}: A \rightarrow \mathcal{P} X$ a function, and $(X, \leq)$ a complete lattice.

Furthermore, from Section 2 we conclude that, together with Top, all the other categories in Table 4 are weakly locally cartesian closed and their exact completions are locally cartesian closed 
categories; moreover, their categories of equilogical objects, which are equivalent to their regular completions, are quasitoposes that fully embed the original categories.

Concerning four of those categories, we also have adjunctions

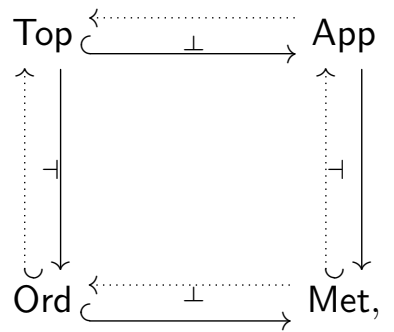

where both solid and dotted diagrams commute, the hook-arrows are full embeddings and the two full embeddings Ord $\hookrightarrow$ App coincide (see [HST14, III-Section 3.6]). One can see that those adjunctions extend to the respective categories of equilogical objects,

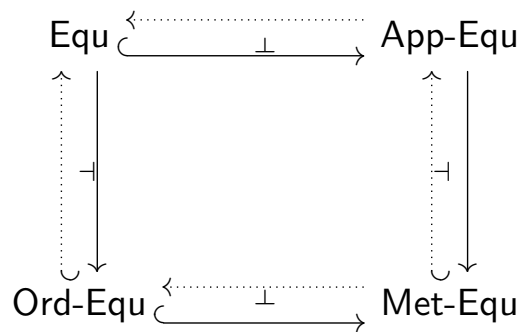

and we describe them now.

(1) Ord-Equ to Met-Equ. Each ordered equilogical object $\left\langle(X, \leq), \equiv_{X}\right\rangle$ is taken to $\left\langle\left(X, d_{\leq}\right), \equiv_{X}\right\rangle$, where the metric $d_{\leq}$is given by

$$
d_{\leq}\left(x, x^{\prime}\right)=\left\{\begin{array}{cl}
0, & \text { if } x \leq x^{\prime} \\
\infty, & \text { otherwise }
\end{array}\right.
$$

for each $x, x^{\prime} \in X$. The left adjoint of this inclusion assigns $\left\langle\left(X, \leq_{d}\right), \equiv_{X}\right\rangle$ to $\left\langle(X, d), \equiv_{X}\right\rangle$, with $x \leq_{d} x^{\prime}$ if and only if $d\left(x, x^{\prime}\right)<\infty$, for each $x, x^{\prime} \in X$. Hence the category Ord-Equ is fully embedded in Met-Equ as the metric equilogical objects $\left\langle(X, d), \equiv_{X}\right\rangle$ for which there exists an order $\leq$ on $X$ such that $d=d_{\leq}$.

(2) Ord-Equ to Equ. Each $\left\langle(X, \leq), \equiv_{X}\right\rangle$ is taken to $\left\langle\left(X, \tau_{\leq}\right)\right.$, $\left.\equiv_{X}\right\rangle$, where $\tau_{\leq}$is the Alexandroff topology: open sets are generated by the down-sets $\downarrow x, x \in X$. For its right adjoint, to an equilogical space $\left\langle(X, \tau), \equiv_{X}\right\rangle$ is assigned $\left\langle\left(X, \leq_{\tau}\right), \equiv_{X}\right\rangle$, where $\leq_{\tau}$ is the specialization order of $(X, \tau)$ : for each $x, x^{\prime} \in X, x \leq x^{\prime}$ if and only if $\dot{x} \rightarrow x^{\prime}$, where $\dot{x}$ denotes the principal ultrafilter on $x$, and $\rightarrow$ denotes the convergence relation between ultrafilters and points of $X$ determined by $\tau$; observe that this is the induced order described in item (a) above. Hence the category Ord-Equ is fully embedded in Equ as the equilogical spaces $\left\langle(X, \tau), \equiv_{X}\right\rangle$ for which there exists an order $\leq$ on $X$ such that $\tau=\tau_{\leq}$, and those are exactly the Alexandroff spaces: arbitrary intersections of open sets are open (see [HST14, II-Example 5.10.5, III-Example 3.4.3(1)]). 
(3) Met-Equ to App-Equ. A metric equilogical object $\left\langle(X, d), \equiv_{X}\right\rangle$ becomes an approach equilogical one $\left\langle\left(X, \delta_{d}\right), \equiv_{X}\right\rangle$, where the approach distance is given by $\delta_{d}\left(x^{\prime}, A\right)=\inf \left\{d\left(x, x^{\prime}\right) \mid x \in A\right\}$, for each $x^{\prime} \in X, A \in \mathcal{P} X$ [HST14, III-Examples 2.4.1(1)]. The right adjoint of this embedding assigns $\left\langle\left(X, d_{\delta}\right), \equiv_{X}\right\rangle$ to $\left\langle(X, \delta), \equiv_{X}\right\rangle$, where $d_{\delta}\left(x, x^{\prime}\right)=\sup \left\{\delta\left(x^{\prime}, A\right) \mid x \in A \in \mathcal{P} X\right\}$, for each $x, x^{\prime} \in X$. Hence Met-Equ is identified within App-Equ as the approach equilogical objects $\left\langle(X, \delta), \equiv_{X}\right\rangle$ such that $\delta=\delta_{d}$, for some metric $d$ on $X$, that is, $(X, \delta)$ is a metric approach space [Low97, Chapter 3].

(4) Equ to App-Equ. Each equilogical space $\left\langle(X, \tau), \equiv_{X}\right\rangle$ becomes an approach equilogical one $\left\langle\left(X, \delta_{\tau}\right), \equiv_{X}\right\rangle$ with the approach distance given by

$$
\delta_{\tau}\left(x^{\prime}, A\right)=\left\{\begin{aligned}
0, & \text { if } A \in \mathfrak{x}, \text { for some } \mathfrak{x} \in U X \text { with } \mathfrak{x} \rightarrow x^{\prime} \\
\infty, & \text { otherwise }
\end{aligned}\right.
$$

for each $x^{\prime} \in X, A \in \mathcal{P} X$, where $U X$ denotes the set of ultrafilters on $X$ HST14, III-Examples 2.4.1(2)]. The left adjoint of this embedding is slightly more elaborate: for an approach equilogical object $\left\langle(X, \delta), \equiv_{X}\right\rangle$, consider the convergence relation $\rightarrow$ between ultrafilters in $U X$ and points of $X$ given by

$$
\mathfrak{x} \rightarrow x \Longleftrightarrow \sup \{\delta(x, A) \mid A \in \mathfrak{x}\}<\infty
$$

this convergence defines a pseudo-topological space [Cho48], to which we apply the reflector described in [HST14, III-Exercise 3.D], obtaining an equilogical space $\left\langle\left(X, \tau_{\delta}\right), \equiv_{X}\right\rangle$. Hence Equ is identified within App-Equ as the approach equilogical objects $\left\langle(X, \delta), \equiv_{X}\right\rangle$ such that $\delta=\delta_{\tau}$, for some topology $\tau$ on $X$, that is, $(X, \delta)$ is a topological approach space [Low97, Chapter 2].

Open question. The conditions (a) to (f) of Sections 1 and 2 were derived from the successful attempt of generalizing the structures/constructions to $(\mathbb{T}, \mathrm{V})$-Cat, for suitable $\mathbb{T}$ and $\mathrm{V}$. Requiring those conditions on an arbitrary category with a topological functor over Set produced the same desired results. However, we do not know an example of a category satisfying those conditions which cannot be described as $(\mathbb{T}, \mathrm{V})$-Cat.

\section{ACKNOWLEDGMENTS}

This work was done during the preparation of the author's $\mathrm{PhD}$ thesis, under the supervision of Maria Manuel Clementino, whom the author thanks for proposing the investigation and advising the whole study. I also thank Fernando Lucatelli Nunes for fruitful discussions.

\section{REFERENCES}

[AHS90] Jiří Adámek, Horst Herrlich, and George E. Strecker. Abstract and concrete categories. Pure and Applied Mathematics (New York). John Wiley \& Sons, Inc., New York, 1990. The joy of cats, A Wiley-Interscience Publication.

[BBS04] Andrej Bauer, Lars Birkedal, and Dana S. Scott. Equilogical spaces. Theoret. Comput. Sci., 315(1):35-59, 2004 . 
[BCRS98] Lars Birkedal, Aurelio Carboni, Giuseppe Rosolini, and Dana S. Scott. Type theory via exact categories (extended abstract). In Thirteenth Annual IEEE Symposium on Logic in Computer Science (Indianapolis, IN, 1998), pages 188-198. IEEE Computer Soc., Los Alamitos, CA, 1998.

[Car95] Aurelio Carboni. Some free constructions in realizability and proof theory. J. Pure Appl. Algebra, 103(2):117-148, 1995.

[CCH15] Dimitri Chikhladze, Maria Manuel Clementino, and Dirk Hofmann. Representable (T, V)-categories. Appl. Categ. Structures, 23(6):829-858, 2015.

[CH03] Maria Manuel Clementino and Dirk Hofmann. Topological features of lax algebras. Appl. Categ. Structures, 11(3):267-286, 2003.

[CH04] Maria Manuel Clementino and Dirk Hofmann. On extensions of lax monads. Theory Appl. Categ., 13:No. 3, 41-60, 2004.

[CH09] Maria Manuel Clementino and Dirk Hofmann. Lawvere completeness in topology. Appl. Categ. Structures, 17(2):175-210, 2009.

[CHJ14] Maria Manuel Clementino, Dirk Hofmann, and George Janelidze. The monads of classical algebra are seldom weakly Cartesian. J. Homotopy Relat. Struct., 9(1):175-197, 2014.

[Cho48] Gustave Choquet. Convergences. Ann. Univ. Grenoble. Sect. Sci. Math. Phys. (N.S.), 23:57-112, 1948.

[CHR18] Maria Manuel Clementino, Dirk Hofmann, and Willian Ribeiro. Cartesian closed exact completions in topology. Preprint 18-46, Dept. Mathematics, Univ. Coimbra, arXiv 1811.03993, 2018.

[CM82] Aurelio Carboni and R. Celia Magno. The free exact category on a left exact one. J. Austral. Math. Soc. Ser. A, 33(3):295-301, 1982.

[CR00] Aurelio Carboni and Giuseppe Rosolini. Locally Cartesian closed exact completions. J. Pure Appl. Algebra, 154(1-3):103-116, 2000. Category theory and its applications (Montreal, QC, 1997).

[CT03] Maria Manuel Clementino and Walter Tholen. Metric, topology and multicategory - a common approach. J. Pure Appl. Algebra, 179(1-2):13-47, 2003.

[CT14] Maria Manuel Clementino and Walter Tholen. From lax monad extensions to topological theories. In Categorical methods in algebra and topology, volume 46 of Textos Mat./Math. Texts, pages 99-123. Univ. Coimbra, Coimbra, 2014.

[CV98] Aurelio Carboni and Enrico M. Vitale. Regular and exact completions. J. Pure Appl. Algebra, 125(1-3):79116, 1998.

[CVO17] Eva Colebunders and Karen Van Opdenbosch. Topological properties of non-Archimedean approach spaces. Theory Appl. Categ., 32:Paper No. 41, 1454-1484, 2017.

[Day72] Brian Day. A reflection theorem for closed categories. J. Pure Appl. Algebra, 2(1):1-11, 1972.

[GHK+80] Gerhard Gierz, Karl Heinrich Hofmann, Klaus Keimel, Jimmie D. Lawson, Michael W. Mislove, and Dana S. Scott. A compendium of continuous lattices. Springer-Verlag, Berlin-New York, 1980.

[Hof07] Dirk Hofmann. Topological theories and closed objects. Adv. Math., 215(2):789-824, 2007.

[Hof11] Dirk Hofmann. Injective spaces via adjunction. J. Pure Appl. Algebra, 215(3):283-302, 2011.

[Hof14] Dirk Hofmann. The enriched Vietoris monad on representable spaces. J. Pure Appl. Algebra, 218(12):2274$2318,2014$.

[HR13] Dirk Hofmann and Carla D. Reis. Probabilistic metric spaces as enriched categories. Fuzzy Sets and Systems, 210:1-21, 2013.

[HST14] Dirk Hofmann, Gavin J. Seal, and Walter Tholen, editors. Monoidal topology, volume 153 of Encyclopedia of Mathematics and its Applications. Cambridge University Press, Cambridge, 2014. A categorical approach to order, metric, and topology. 
[Law02] F. William Lawvere. Metric spaces, generalized logic, and closed categories [Rend. Sem. Mat. Fis. Milano 43 (1973), 135-166 (1974); MR0352214 (50 \#4701)], 2002. With an author commentary: Enriched categories in the logic of geometry and analysis.

[Low97] Robert Lowen. Approach spaces. Oxford Mathematical Monographs. The Clarendon Press, Oxford University Press, New York, 1997. The missing link in the topology-uniformity-metric triad, Oxford Science Publications.

[Man02] Ernest G. Manes. Taut monads and T0-spaces. Theoret. Comput. Sci., 275(1-2):79-109, 2002.

[Men00] Matías Menni. Exact completions and toposes. PhD thesis, University of Edinburgh, 2000.

[MST06] Mojgan Mahmoudi, Christoph Schubert, and Walter Tholen. Universality of coproducts in categories of lax algebras. Appl. Categ. Structures, 14(3):243-249, 2006.

[Ros98] Giuseppe Rosolini. Equilogical spaces and filter spaces, manuscript, 1998.

[Ros99] Jiří Rosický. Cartesian closed exact completions. J. Pure Appl. Algebra, 142(3):261-270, 1999.

[Sch84] Friedhelm Schwarz. Product compatible reflectors and exponentiability. In Categorical topology (Toledo, Ohio, 1983), volume 5 of Sigma Ser. Pure Math., pages 505-522. Heldermann, Berlin, 1984.

[Sco96] Dana S. Scott. A new category? domains, spaces and equivalence relations, manuscript, 1996.

CMUC, Department of Mathematics, University of Coimbra, 3001-501 Coimbra, Portugal

E-mail address: willian.ribeiro.vs@gmail.com 